\title{
Heating of staircases in high-rise buildings
}

\author{
Dmitry Ivanov $^{1}$, Olga Yakimchuk ${ }^{1}$, Ivan Pastukhov ${ }^{2}$ \\ ${ }^{1}$ APEX project bureau, Moscow, Russian Federation \\ ${ }^{2}$ Sberbank Real Estate, Moscow, Russian Federation
}

\begin{abstract}
This study aims to develop recommendations for the heating of staircases in high-rise buildings. In particular, the optimal distribution of convectors on different floors and their heating power output is to be identified. We need to analyze the data from a large number of staircases with various numbers of floors, values of glazing ratio and arrangements of heating units in order to achieve the above-mentioned goal. Conducting field experiments, in this case, is limited to available types of buildings, engineering designs, and weather conditions. We use computational fluid dynamics (CFD) simulations calibrated on a number of field experiments in order to overcome these limitations and obtain the distribution of temperature for all the studied staircases. We additionally do a sensitivity study of various factors such as infiltration rate, heat exchange with adjacent spaces and outdoor air temperature. An engineering methodology based on regression analysis was developed for determining the optimal arrangement of heating units within the staircases.
\end{abstract}

\section{Introduction}

The construction of high-rise buildings has become a global trend due to the lack of space in cities. Residential and public buildings of 25 floors and higher are common nowadays. Considering modern construction scales, the global construction industry requires the development of guidelines for high-rise buildings. Moscow ranks first among the 85 regions of the Russian Federation with a $22.5 \%$ share of the high-rise construction. The majority of buildings under construction in Moscow are higher than 13 floors. Due to the cold climate, energy-efficient heating of such buildings is not trivial. Recommendations for the positioning of the terminal heating units in residential and public areas of high-rise buildings are well defined, which is not the case for the stairs because the heated volume of staircases directly depends on the building height. Capital costs of the construction, the appearance of the stairs, the presence of overheated or overcooled zones of the staircase, and consequently, the operating costs of heating depend on the arrangement of heating devices in the staircases.

The Russian building code contains only one recommendation regarding this issue: "Heating devices on staircases should, as a rule, be placed on the first floor, and on staircases divided into compartments, at the bottom of each compartment" (SP 60.13330.2012, 2012). Thus, the problem of placing the heating devices in the stairs of high-rise buildings is not obvious and does not have a clear solution. As a rule, engineers rely on experience, which varies greatly from person to person, which often leads to completely different design solutions. To verify the recommendation regarding the installation of heating devices in the lower part of the building, numerical modeling of a staircase in a 24-story building with $25 \%$ glazing was carried out with the arrangement of convectors on the first six floors with half of the total power assigned to the first floor. The results showed that the air in the upper nine floors of the stairs is cooler than $13^{\circ} \mathrm{C}$. The situation is similar for a 12 -story building with $50 \%$ glazing and three heating devices located on the first three floors (see Figure 1).

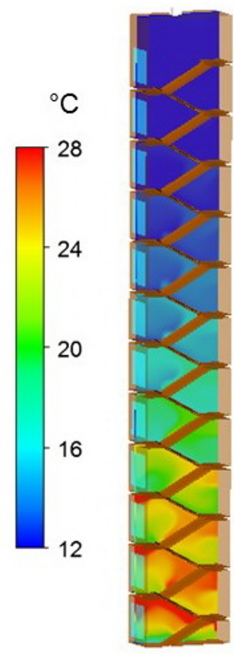

Figure 1: Temperature distribution in the 12-story building staircase (CFD).

This confirms the insufficiency of existing guidelines and methods for determining the optimal placement of the heating units, which leads to the placement of convectors more densely than it is required, or to overcool and overheat of the staircase zones.

Rock (2017) studied heating and cooling of the five-story tall staircase with fan coil units using CFD simulation. The study challenged the rule-of-thumb regarding the sufficiency of a single heating unit at the bottom a stair tower, highlighted the danger of freezing of building services containing water. 
Mokhtarzadeh-Dehghan (2011) concluded that numerical solutions can provide accurate results for the problem and highlighted the importance of thermal radiation in the simulation. Peppes (2001) found a very good agreement between the CFD simulations and experimental measurements for the two-story staircase.

Overall, the literature review revealed very little data on the temperature distribution between the floors of the stairwells and no fixed recommendations on the optimal placement of the heating units. This study was conducted to fill the gap.

\section{Methods}

The following measures were used in order to reach the goal of this study: CFD analysis, field measurement of temperature distribution in stairs of several existing buildings, regression model. These methods are described in details below.

\section{Numerical Simulation Description}

Computational Fluid Dynamics (CFD) was used for numerical modelling of hydrodynamic processes. A CFD model is a combination of a geometric model of a room, a physical model of the processes under consideration, and a mathematical model. The physical model, in turn, includes the properties of air, the parameters of the heating unit operation, the conditions of heat exchange with the environment, infiltration, and other phenomena described in detail below. Taking into account the peculiarities of the physics of the problem and the geometry of space, a mathematical model was set up. The model is a system of equations describing the change of parameters in space and in time in case of the timedependent problem. Its solution allowed us to obtain the distribution of the desired parameters and analyse them. We use ANSYS CFX software for the simulation, which realizes the numerical solution of the heat and mass transfer equations by the finite volume method. Analysis of the results is possible when obtaining the values of the variables of interest - density, pressure, velocity components, temperature. They are related to each other by the Navier-Stokes system of equations of a viscous compressible fluid.

For a more accurate solution to this problem, it is necessary to take into account the flow turbulence. This complex three-dimensional nonstationary phenomenon is the fluctuation of environmental parameters in space and time. At present, there is a large number of turbulence models developed, the choice is determined by the specifics of the problem. For this study, we tested several models, with the result that the standard " $k \varepsilon$ " configuration has proven itself well. Radiation heat transfer was taken into account by the "Discrete Transfer Model" method, which assumes isotropic scattering that is uniform in all directions. This approach is justified in this case due to the absence of a dedicated radiation direction. The finite volume method is used in most software systems to simulate the flow of a liquid or gas.
An important stage of modelling with this method is the creation of a finite element mesh, which is a set of elementary volumes, for which the equations are solved. The accuracy of the model, calculation time and, as a result, the accuracy of the calculation results depend on the number of elements, the shape, and regularity of the grid. We used prismatic elements for more accurate accounting of heat exchange and resolution of the flow near the surfaces of walls, windows and heating devices, convectors in this case., This allowed a small grid size near the wall. For the rest of the volume, a tetrahedral grid was constructed, with higher density around the objects with smaller linear dimensions, as shown in Figure 2.

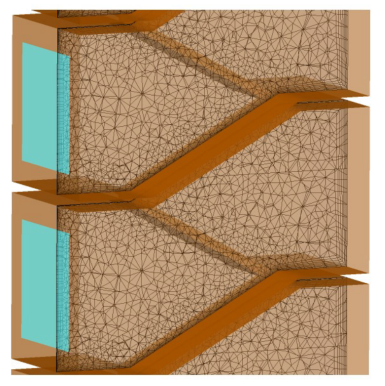

Figure 2: Simulation grid.

Our research was not intended to study the change in air temperature over time. In this case, the solution can be carried out in a steady state, when an iterative method is applied to the system of equations. The solution was stopped when the level of residuals descended below $10^{-4}$ and there was no change in average temperatures across the floors.

The air properties inside the room can vary due to the possible variety of architectural stair solutions and places of convectors installation. In this paper, we chose the most typical construction as an option. The dimensions are present in Figure 3.

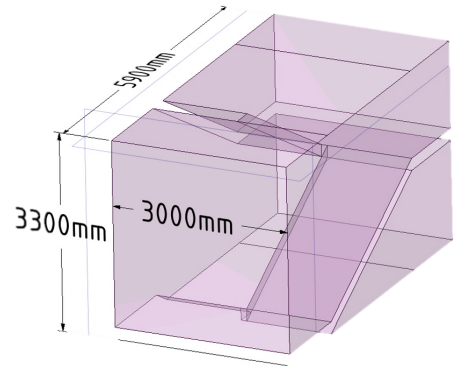

Figure 3: Geometry of the staircase.

A flight of stairs has one external wall with a window, the size of which varies. Glazing area in various experiments ranges from $0 \%$ to $100 \%$. Heat loss through external walls is taken into account with the heat transfer coefficient and a set of outdoor air temperature. We consider the remaining walls internal and take into account the heat exchange through them with adjacent rooms. The average temperature of the coldest five-days with $92 \%$ cumulative frequency for Moscow is $-25^{\circ} \mathrm{C}$; however, for calculating more extreme conditions, the simulation was performed with an outdoor air temperature of $-28^{\circ} \mathrm{C}$. 
In adjacent rooms, the minimum allowable average temperature of $18^{\circ} \mathrm{C}$ for corridors, living rooms, kitchens was selected (according to GOST 30494-2011, 2011). The values of heat transfer coefficients are given in Table 1.

Table 1: Boundary conditions for walls and windows.

\begin{tabular}{|c|c|c|}
\hline Surface & $\begin{array}{c}\text { Heat transfer } \\
\text { coefficient, } \\
\mathbf{W} / \mathbf{m}^{2} \mathbf{K}\end{array}$ & $\begin{array}{c}\text { External } \\
\text { temperature, }\end{array}$ \\
${ }^{\circ} \mathbf{C}$ \\
\hline Exterior wall & 0.33 & -28 \\
\hline Windows & 1.67 & -28 \\
\hline Interior wall & 1 & 18 \\
\hline
\end{tabular}

We placed convectors on the sidewall at a height of $2.2 \mathrm{~m}$ above the floor in accordance with the requirements of fire safety. The heat source is taken into account as a volume energy release; herewith the convective and radiation components of heat transfer are divided in a proportion of $80 \%$ and $20 \%$, respectively, and remain at this level regardless of the temperature around the device.

To evaluate the results of the calculations, it is necessary to determine the criteria for the success of the heating devices placement. Hereinafter we consider that for the comfort of people, the average temperature on any floor should be no more than $2^{\circ} \mathrm{C}$ below the average allowable, thus it should be above $14^{\circ} \mathrm{C}$. In addition, an important criterion is the freezing of structures, in particular, glazing. The minimum temperature of the inner surface of the glazing should not be below $3^{\circ} \mathrm{C}$. However, performed calculations showed that the inner surface of the windows with heat transfer coefficient specified in Table 1 would not cool below $5^{\circ} \mathrm{C}$ provided an average air temperature at each floor be above $14^{\circ} \mathrm{C}$. Thus this criterion is satisfied automatically and does not require additional verification.

\section{Calibration of a numerical model with the field experiments}

Computer calculations need to be compared with existing databases, test cases, or experiments, before concluding about the results. Within the framework of this study, field experiments were carried out that made it possible to evaluate the validity of the simulations.

The air temperature measurements in the stairwells were carried out in five various types of buildings in winter, along with the measurement of outdoor air temperatures, wall temperatures with an infrared pyrometer, and heating loads on every floor. Table 2 presents the characteristics of these buildings and the results of the measurements in the form of minimum $\left(T_{\min }\right)$, maximum $\left(T_{\max }\right)$ and average
( $\left.T_{\text {ave }}\right)$ temperatures, indicating the external $\left(T_{\text {ext }}\right)$ temperature during experiments.

These experiments showed overheating of the staircase relative to the optimal values in all measurement cases. Average temperature values exceeded the allowable range in three of five buildings. Taking into account the different types of buildings considered, it can be concluded that the heating system is inefficient and the power consumption is excessive.

Described experiments were reproduced in CFD to calibrate a numerical model and verify the physical assumptions and the mathematical model. We built geometries of all measured stairs with the features of the structures and weather conditions taken into account. Heat transfer coefficients in each case correspond to the available data. Comparison of the temperatures obtained in the field and numerical experiments made it possible to select the most important factors. On average the temperature error was less than $5 \%$ relative to the experimental data. Below we discuss the factors that have the greatest impact:

- gravity (buoyancy), which plays a huge role in nonisothermal processes;

- heat exchange with the exterior, which determines the main source of heat loss;

- glazing ratio - the more the window area, the more the heat loss on each floor and the more often it is necessary to arrange the devices;

- power and location of heating devices;

- main structural elements, such as exterior and interior walls, windows, flights of stairs.

Note that it is advisable to simplify the geometry and exclude objects that have little effect on air flow, but require serious increases in computational resources due to the small linear dimensions and the need to thicken the grid at their locations. Such objects include, for example, railings, window and door handles, window frames, protrusions on the walls, steps, decorative elements.

Other factors are less obvious and their significance is determined after carrying out a series of simulations. Varying the processes taken into account, analysing the simulation results and comparing it with experimental data showed the importance of the following parameters:

- infiltration;

- heat exchange with adjacent rooms;

- outdoor temperature;

- radiation heat transfer.

Table 2: Specification of the buildings for field experiments and the temperatures obtained.

\begin{tabular}{|c|c|c|c|c|c|c|c|c|}
\hline$\#$ & $\begin{array}{c}\text { Year of } \\
\text { construction }\end{array}$ & $\begin{array}{c}\text { Number } \\
\text { of floors }\end{array}$ & $\begin{array}{c}\text { Number of heating } \\
\text { units }\end{array}$ & $\begin{array}{c}\text { Glazing } \\
\text { ratio, } \%\end{array}$ & $\boldsymbol{T}_{\boldsymbol{e x t}}{ }^{\circ} \mathbf{C}$ & $\boldsymbol{T}_{\boldsymbol{m i n},}{ }^{\circ} \mathbf{C}$ & $\boldsymbol{T}_{\boldsymbol{m a x}},{ }^{\circ} \mathbf{C}$ & $\boldsymbol{T}_{\boldsymbol{a} \text { ave, }}{ }^{\circ} \mathbf{C}$ \\
\hline 1 & 1962 & 5 & 2 & 15 & -18 & 14 & 22 & 18.6 \\
\hline 2 & 1985 & 17 & 12 & 20 & -7 & 16 & 22 & 20.1 \\
\hline 3 & 2002 & 14 & 13 & 10 & -8 & 16 & 21 & 19.3 \\
\hline 4 & 2016 & 17 & 10 & 40 & -2 & 16 & 23 & 21.6 \\
\hline 5 & 2013 & 15 & 14 & 44 & -2 & 20 & 23 & 22.2 \\
\hline
\end{tabular}


In the article, we research in more details the infiltration as the most sophisticated and interesting factor. In the results section, we show that the catalogued infiltration values of the constructions were underestimated and their usage leads to an unrealistic temperature distribution in the stair volume. A numerical experiment with increased infiltration, on the contrary, is in a good agreement with a full-scale experiment. Thus, we can conclude that infiltration has a great influence on the temperature distribution along the height by increasing the air mixing and its accurate accounting is necessary to obtain the correct simulation results.

\section{Regression model development}

In order to improve the energy efficiency and reduce the number of heating devices, it is necessary to develop a methodology based on the results of numerical modelling and experiments, which will allow efficient heating of stairs.

It is necessary to find a relationship between the factors influencing the temperature distribution in stairs to develop the methodology. The sought dependencies were identified through regression analysis. The definition of regression analysis can be given as follows: this is a statistical method for studying the influence of one or several independent variables on a dependent variable. Independent variables are otherwise called regressors or predictors. At the moment, the regression models are described in sufficient detail and have proven to be a good tool that describes a pattern with the help of a wide class of universal functions. To build the model, the measured data are mainly used; knowledge of the properties of the studied regularity is not mandatory. Thus, after conducting numerous experiments and collecting a database of measured values, one can train a model by finding the optimal set of parameters and coefficients.

In this study, simulation results act as such experiments, the validity of which is proved with the field experiments.

The goal of regression analysis is to find an approximate solution of the true functional dependence. Multiple regression techniques exist, some of which were applied for this study: linear regression, adaptive splines (MARS), decision trees and random forests, neuron network and Naïve Bayes. Linear regression showed the least error at the test sample and will be discussed next.

It was necessary to optimize the experiment on the following factors: number of input parameters, interval, and number of variation levels, the sequence of the experiments. Therefore, it was necessary to define the matrix of experiments in such a way, as to fill in the volume of the variables mostly evenly and to take into account all the parameters, which influence the dependent variable. A fractional factorial experiment was used for this problem.

Total number of 38 experiments were conducted with following varying parameters: number of floors (from 8 to 36$)$, glazing ratio $(0,10,25,50$ and 100 percent), number of heating units and their placement. First 18 experiments were conducted in order to even distribution of the parameters; the following 20 simulations were aimed at filling the most appropriate intervals to meet the criteria for success.

Table 3: Numerical simulation experiments.

\begin{tabular}{|c|c|c|c|}
\hline$\#$ & Floors & $\begin{array}{c}\text { Glazing } \\
\text { ratio, } \%\end{array}$ & $\begin{array}{c}\text { Number of } \\
\text { heating } \\
\text { units }\end{array}$ \\
\hline 1 & 36 & 100 & 3 \\
\hline 2 & 36 & 0 & 6 \\
\hline 3 & 36 & 50 & 12 \\
\hline 4 & 24 & 0 & 2 \\
\hline 5 & 24 & 50 & 4 \\
\hline 6 & 24 & 100 & 8 \\
\hline 7 & 12 & 50 & 1 \\
\hline 8 & 12 & 100 & 2 \\
\hline 9 & 12 & 0 & 4 \\
\hline 10 & 32 & 75 & 4 \\
\hline 11 & 32 & 10 & 8 \\
\hline 12 & 32 & 25 & 16 \\
\hline 13 & 16 & 10 & 2 \\
\hline 14 & 16 & 25 & 4 \\
\hline 15 & 16 & 75 & 8 \\
\hline 16 & 8 & 25 & 1 \\
\hline 17 & 8 & 75 & 2 \\
\hline 18 & 8 & 10 & 4 \\
\hline 19 & 25 & 25 & 5 \\
\hline 20 & 25 & 50 & 5 \\
\hline 21 & 25 & 100 & 6 \\
\hline 22 & 36 & 0 & 2 \\
\hline 23 & 36 & 50 & 8 \\
\hline 24 & 36 & 50 & 8 \\
\hline 25 & 24 & 0 & 2 \\
\hline 26 & 24 & 50 & 5 \\
\hline 27 & 24 & 100 & 7 \\
\hline 28 & 12 & 50 & 3 \\
\hline 29 & 12 & 100 & 3 \\
\hline 30 & 12 & 0 & 1 \\
\hline 31 & 36 & 100 & 10 \\
\hline 32 & 32 & 75 & 7 \\
\hline 33 & 32 & 10 & 4 \\
\hline 34 & 32 & 25 & 5 \\
\hline 35 & 16 & 10 & 2 \\
\hline 36 & 16 & 25 & 2 \\
\hline 37 & 16 & 75 & 4 \\
\hline 38 & 8 & 10 & 1 \\
\hline
\end{tabular}

The results of simulations were analysed pertaining to the heating units, so the total number of 181 points, for which the parameters of the problem (glazing ratio, floor of the installation, heating output, infiltration, heat flow from the internal walls and others), as well as the simulation results (minimum, average and maximum temperature in zone served by the unit).

If the sough parameter depends on the multiple variables, then this regression is called multivariable. In such a case, a question of the necessity of including all the available parameters in the regression model arises. An excessive number of parameters may lead to overtraining of the model. In order to reduce the number of independent variables, an analysis of the importance of parameters was carried out. We analysed the following as a dependent parameter: the minimal average temperature in the served 
zone, number of floors, which can be heated by the unit, the satisfaction of success criteria. The first one showed to be the most convenient in use.

To create the model, among the directly obtained parameters, additional universal parameters that describe the values of certain general characteristics were used. For instance, the relative installation height of the unit varies from 0 to 1 and is recalculated depending on the building height in such a way as 0 relates to the first floor and 1 to the last. Such an approach allowed finding general dependencies leading to higher model accuracy. The following section contains the results of the study.

\section{Results}

This section consists of the discussion on the realistic values of infiltration in the field experiments, the importance of its correct determination for the simulations and the results of regression analysis.

\section{Infiltration and its impact on temperature distribution}

Infiltration is the penetration of outside air into the building. This is possible due to the existing leaks and gaps, opening windows and doors. Pressure difference on opposite sides of the gap is the cause of air movement. There are many studies showing a significant contribution of infiltration to the buildings heat loss in winter and heat gain in summer, despite the use of modern materials and weatherstripped windows. (Younes, 2011, Persily, 1999)

The need to account for infiltration is described in the standards. Methods to assess the amount of incoming air can be found in the textbooks of various authors, e.g. Malyavina (2007). It allows calculating the infiltration on each floor separately, depending on the pressure difference, type of the windows and entrance doors used. The largest infiltration values correspond to the lower floors, it decreases as they rise up, reaching zero values in the middle of the stair's height. At the same time in the upper part of the building, internal air is exfiltrated.

Unobvious questions are how to properly consider this process and calculate its influence. To simplify the mathematical model, there is a temptation to set this load not through the inflow of cold air, but as additional heat loss from the wall surfaces or windows. However, the analysis of simulation results with different boundary conditions showed the importance of taking into account the infiltrating air in an explicit form, because it contributes to the effect of the warm air rising up.

Let us consider an example of an infiltration impact on the temperature distribution of the staircase. Air temperature measurements were conducted in a five-story building in winter. Numerical simulations were performed for the same staircase with various infiltration rates: normalized and increased over the first one. Figure 4 shows a comparison of the obtained results with the experiment.

As can be seen, low infiltration values (or its complete absence) lead to unrealistic temperature distribution, namely to the accumulation of warm air at the bottom of the volume. Rock (2017) shows a similar picture. Unfortunately, their article does not describe in detail the used boundary conditions; presumably, they applied small infiltration rate or did not include it as an airflow rate. On the contrary, a numerical simulation with increased infiltration fits the experiment well. Thus, we conclude that infiltration has a great influence on the temperature distribution by increasing the mixing of the air. Its accurate accounting is necessary to obtain the correct simulation results.

\section{Actual infiltration values}

In SP 50.13330.2012 (2012) "the amount of infiltrating air entering the stairwell of a residential building or into a public building through the leak-tightness of filling openings" is determined by the equation (1).

$$
G_{\text {inf }}=\left(\frac{S_{w}}{R_{w}}\right) \cdot\left(\frac{\Delta p_{w}}{10}\right)^{2 / 3}+\left(\frac{S_{d}}{R_{d}}\right) \cdot\left(\frac{\Delta p_{d}}{10}\right)^{1 / 2}
$$

Where $S_{w}$ and $S_{d}$ are the windows and eternal doors total areas, $R_{w}$ and $R_{d}$ are air penetration resistances for windows and external doors and $\Delta p_{w}$ and $\Delta p_{d}$ are the calculated pressure differences between the external and internal air on windows and external doors respectively.

The pressure difference, and accordingly, the required resistance to air penetration, depends on the height of the building. The higher the building, the greater the pressure drop and $R_{w}$ or $R_{d}$.
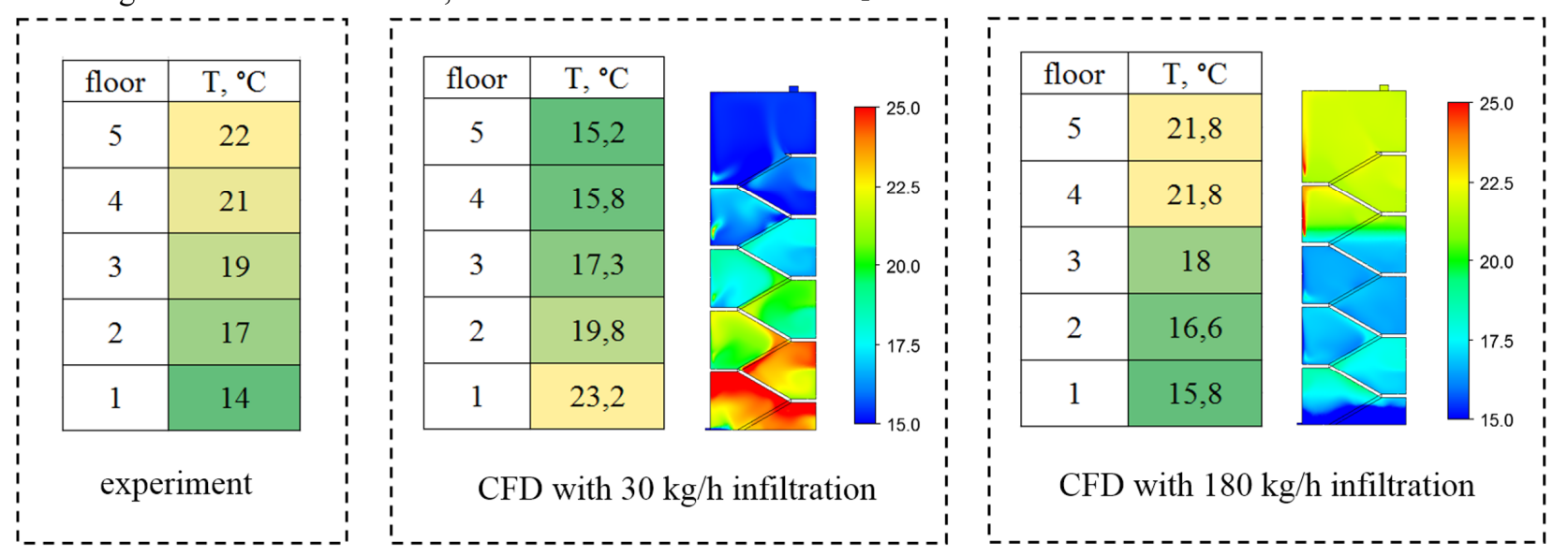

Figure 4: Results of the experiment and simulation at different infiltration intensity in the 5-story building. 
Thus, having determined the resistance to air penetration of windows and doors, knowing their location and area, building height, wind speed, it is possible to find the flow rate of infiltration air on each floor according to Malyavina (2007). With this approach, there was a discrepancy in the temperatures obtained in numerical calculations with experimental data. Therefore, it was required to select other values for resistance to air penetration, which ensured realistic air circulation and correct temperature distribution. An analysis of Russian literature did not reveal recommendations for increased infiltration with respect to the normalized values or methods for accounting the amount of actually flowing air in a building through leaks. In the foreign literature, there are a number of published studies on the penetration of air into the building. In particular, the technical note of Orme (1994) aims to summarize the available data on the transmission of air by building structures for use in calculations. The authors created a computer database compiled from information found in published sources and obtained the values of elements crack flow coefficients. They grouped them according to the frequency of propagation, that is, by quartiles.

The lower quartile means that a quarter of all objects have values of the required coefficient less than the specified one. The median shows such a coefficient, in relation to which half of all objects have values less than it. Median is considered an indicator of good quality material. The number reflecting such a value of the coefficient at which the value in a real object will be lower than that specified with a probability of $75 \%$ is called the upper quartile. It reflects the poor quality of the design. As stated in the study, most of the standards regulate the low penetration of the elements and correspond to the lower quartile in the table. If there are no data on experimental measurement of coefficients, then a recommendation is given to rely on median values. So there is the need to increase the calculated infiltration relative to the value required by the norms in order to obtain data corresponding to the experiments.

According to the above-mentioned method, selected resistance coefficients for air penetration were recalculated for the five field experiments described above. The obtained figures show how the actual infiltration of air exceeds the calculated according to the norms. In all experiments, the air penetration resistance values are no less than 7 times lower than the calculated ones, except for the last case in which this coefficient for windows corresponds to normalized ones. Figures 5 and 6 illustrate the boundaries of quartiles of weatherstripped and non-weatherstripped windows and doors. Also, the coefficients obtained from field experiments are marked with symbols on the chart. One can see that almost all points for windows lie within the upper quartile of the weatherstripped windows and the lower quartile of the non-weatherstripped. Similarly, Figure 6 shows the correspondence of external doors to the upper quartile of the non-weatherstripped doors. Point number 3 is the outlier and it can be concluded that the door of this building is not close tightly and does not fulfil its function.

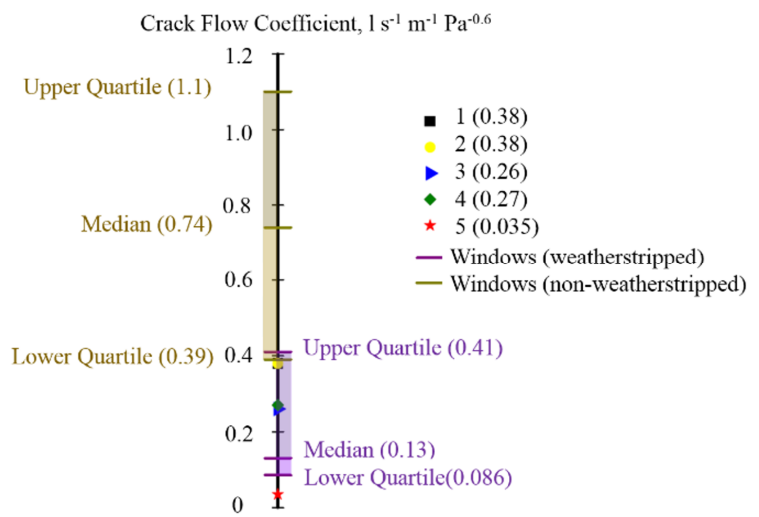

Figure 5: Crack flow coefficient for windows.

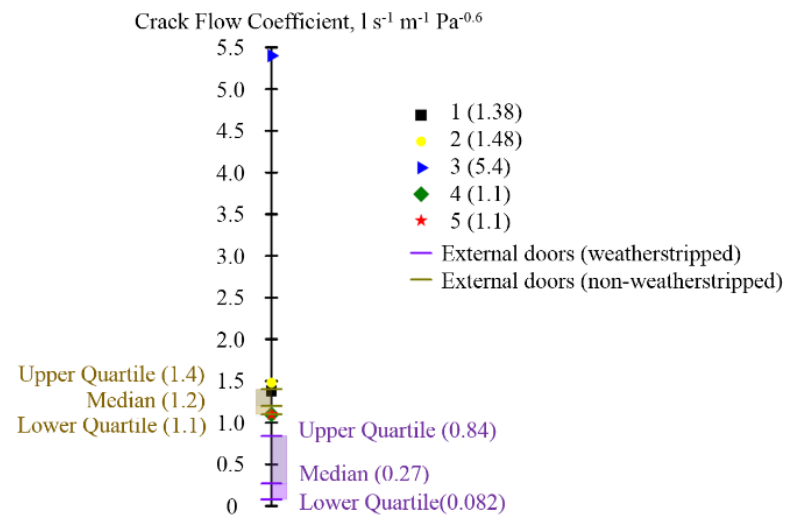

Figure 6: Crack flow coefficient for doors.

We have proposed the following method for determining infiltration in buildings under construction, used in this study. It is necessary to choose the median values of the air crack flow coefficient for weatherstripped windows $\left(0.13 \mathrm{ls}^{-1} \mathrm{~m}^{-1} \mathrm{~Pa}^{-0.6}\right)$ and non-weatherstripped doors $\left(1.2 \mathrm{ls}^{-1} \mathrm{~m}^{-1} \mathrm{~Pa}^{-0.6}\right)$. Further, for each separately considered staircase, it is necessary to recalculate the resistance to air penetration according to the equation (2).

$$
R=\frac{1}{C} \cdot \frac{S}{L} \cdot \frac{\Delta p^{1 / 15}}{3.6 \cdot 10^{2 / 3 \cdot 1.2^{0.5} \cdot \rho^{0.5}}}
$$

Where $C$ is a crack flow coefficient, $S$ is the area of an element, $L$ is its perimeter, and $\rho$ is the density of the inflowing air.

\section{Regression model and its accuracy}

The obtained regression model that predicts the minimum average air temperature of the floor in the zone served by the unit can be represented in the following form:

$$
y=a_{0}+\sum_{i=1}^{9} a_{i} \cdot x_{i}
$$

for which selected regressors $x_{i}$ and the values of regression coefficients $a_{i}$ are shown in Table 4 .

We randomly selected 31 of 181 points for model testing. The training sample consisted of 150 points and the root mean square error of the predictions in it was $0.44^{\circ} \mathrm{C}$. Same value for the test sample was $0.53^{\circ} \mathrm{C}$. The 
coefficient of variation was $3 \%$ for the training sample and $3.6 \%$ for the test sample.

Table 4: Regression model parameters.

\begin{tabular}{|c|c|c|}
\hline $\boldsymbol{x}_{\boldsymbol{i}}$ & Variable & $\boldsymbol{a}_{\boldsymbol{i}}$ \\
\hline & Intercept & 15.575 \\
\hline$x_{1}$ & Glazing ratio & 2.61 \\
\hline$x_{2}$ & Infiltration (W) & -0.0000632 \\
\hline$x_{3}$ & Interior walls (W) & -0.00126 \\
\hline$x_{4}$ & Exterior walls (W) & -0.000508 \\
\hline$x_{5}$ & Windows (W) & 0.000993 \\
\hline$x_{6}$ & Floor of installation & 0.00194 \\
\hline$x_{7}$ & Relative installation height & 1.224 \\
\hline$x_{8}$ & Relative heating power & 0.127 \\
\hline$x_{9}$ & $\begin{array}{c}\text { Heating power to the served } \\
\text { floors (W) }\end{array}$ & -0.000913 \\
\hline
\end{tabular}

The predicted average temperature distribution along the values from the simulation experiments and the distribution of residuals are in Figures 7 and 8.

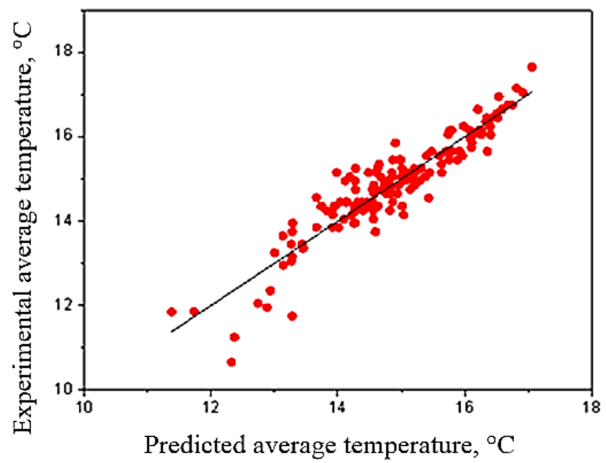

Figure 7: Regression model predicted temperatures

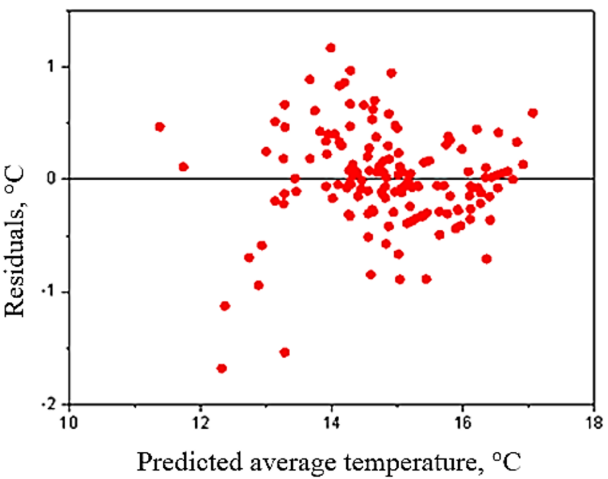

Figure 8: Regression model residuals

Application of the regression model is an iterative process. Firstly, one needs to determine the inputs: glazing ratio, heat gains and loss from infiltration, interior and exterior walls. Heating units are placed from the bottom to the top of a staircase. Then, the heating output of the convector on the first floor is to be calculated, as well as the number of floors it serves. Afterward, the next heating unit is placed at the floor over the served zone of the first convector and its power output is calculated. This procedure repeats until compensating all the heat loss of the staircase. For the sake of usability, the calculation procedure was realized in the Excel file.

In order to verify the methodology, the 30 -floor building with a $100 \%$ glazing ratio was selected. The application of the above-mentioned approach results in 7 heating units. According to the regression model, it is necessary to place the convectors at $1,3,6,9,13,18$ and 24 floors. The CFD simulation of this staircase and the average air temperatures are presented in Figure 9.

The above results indicate that the proposed approach allows calculating the number of heating units and its placement in a way that ensures heating of the staircase volume to the temperature required without waste of costs.

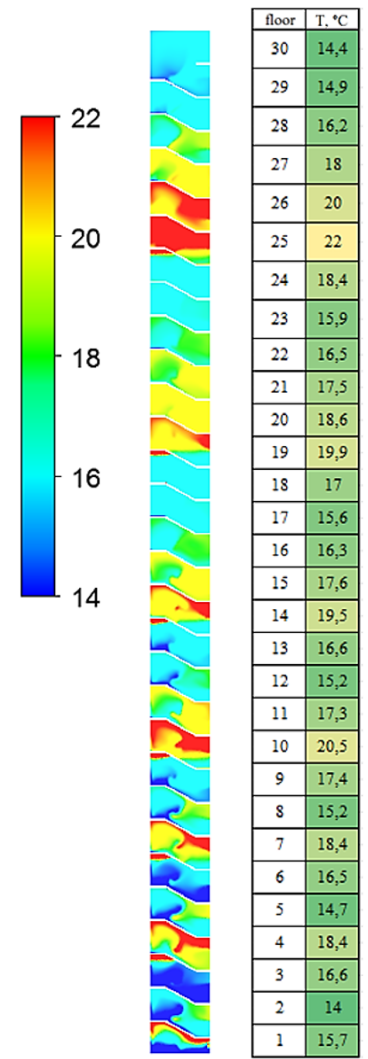

Figure 9: Regression method prediction.

\section{Discussion}

The most accurate evaluation of the placement of heating units in staircases would be CFD simulation of every particular design case. However, it is not always possible or necessary to follow such an approach for typical project designs. For more effective use of resources and reduction of the quantity of heating units, we developed a methodology based on the results of numerical and field experiments.

The proposed methodology is based on a quite detailed calculation of the heat balance of a staircase including the components, which are not always taken into account in engineering practice (such as heat gains from the interior spaces). Also, the proposed approach requires a calculation of infiltration, based on the worse air impermeability properties of constructions, than the ones in the codes or catalogues.

It should be noted that the authors carried out additional simulations with higher outdoor air temperature. The results revealed that the proposed approach is applicable 
in the climate zones with the design outdoor air temperature higher than $-28{ }^{\circ} \mathrm{C}$, resulting in more conservative and suboptimal distribution of heating units.

The application of the methodology showed the fairness of recommendations to place heating units more often at the bottom of the stairs, rather than in the top, where there is no infiltration. After the series of experiments, we made the conclusion that it is enough to place only one heating unit on the first floor in a 30 or fewer floors staircase without external glazing. However, it is worth mentioning that the proposed approach does not guarantee the reproduction of the calculated parameters of the environment due to limits of modelling both in terms of inclusion of all physical processes in the model, as well as limitations of computational resources. It is necessary to ensure the following criteria are in place before the application of the proposed algorithm:

- the building under consideration is located in a climate zone with the design outdoor temperature of $-28^{\circ} \mathrm{C}$ or higher;

- the staircase is a single volume. If it is broken down into zones without free air movement between them, then each one should be considered separately;

- internal and external dimensions of a staircase should resemble such as shown in Figure 3. If the geometry of a staircase is not standard, the methodology should be applied with caution;

- three walls of a staircase should be internal; while the external wall should have less area and may have any glazing ratio;

- the number of floors in staircases for which the methodology has been tested was limited to 40 . In the case of higher staircases, the methodology should be applied with caution.

\section{Conclusion}

Due to the lack of guidelines for heating of stairs in highrise buildings, there is an uncertainty in the placement of heating devices and the problem of using too many of them. Overheating of stairs in winter and inefficient use of resources is also a common problem confirmed by our field experiments. This paper presents a method for determining the optimal number of heating units in standard stairs in order to ensure the greatest efficiency and reducing their number to the minimum necessary. This allows for achieving the desired temperature distribution over the staircase volume. The method is based on a linear regression model built after conducting a series of numerical experiments. We calibrated the numerical models based on the field measurements. In the course of the study, we identified the important factors, consideration of which is necessary for obtaining reliable results. A realistic estimate of the amount of air infiltrating in the staircase volume is critical for obtaining the temperature distribution corresponding to reality.
A new method was proposed for determining the amount of infiltrating air inside the staircase volume because the use of existing recommendations does not lead to obtaining results that correspond to measurements in the existing buildings. The developed methodology allows determining the density and power distribution of convectors by floors. The method performed well when analysing the results of a numerical calculation carried out after determining the placement of heating devices by the regression model and it can be used for designing heating in stairs.

This article focused on the aspect of heating of the staircases, which is more relevant for a cold climate. The future works may address the issues of cooling of the high-rise staircases as well as a more complete generalization for different climates and geometries of staircases.

\section{Acknowledgement}

The authors would like to thank APEX project bureau for the support of the study.

\section{References}

Malyavina, E.G. (2007). Heat losses inside buildings. ABOK-PRESS. Moscow (Russian Federation)

Ministry of Regional Development of Russia (2012). SP 50.13330.2012 Thermal performance of the buildings.

Ministry of Regional Development of Russia (2012). SP 60.13330.2012. Heating, ventilation and conditioning.

Mokhtarzadeh-Dehghan, M. R., (2011) Numerical simulation and comparison with experiment of natural convection between two floors of a building model via a stairwell. Int. J. of Heat and Mass Transfer 54(1-3), 19-33.

Orme, M. (1994). Technical Note 44. An analysis and data summary of the AIVC's numerical database. AIVC. Coventry (UK).

Persily, A. (1999). Myths About Building Envelopes. ASHRAE Journal 41(2), 39-45.

Peppes, A. A., Santamouris M., Asimakopoulos D.N. (2001). Buoyancy driven flow through a stairwell. Building and Environment, 36(2):167-180.

Rock, B.A., McDonald, J.D. (2017) Ups and downs of stair towers: improving comfort and IAQ. ASHRAE Journal 59(12), 12-20.

SantehNIIproekt (2011). GOST 30494-2011. Residential and public buildings. Microclimate parameters for indoor enclosures.

Younes, C., Abi Shdid, C., Bitsuamlak, G. (2011). Air infiltration through building envelopes: A review. Journal of Building Physics 35(3), 267-302. 